Check for updates

Cite this: Phys. Chem. Chem. Phys., $2020,22,818$

Received 14th October 2019, Accepted 4th December 2019

DOI: $10.1039 / c 9 c p 05599 a$

rsc.li/pccp

\title{
Doping homogeneity in co-doped materials investigated at different length scales $\dagger$
}

\author{
Wenyu Li, (D) ${ }^{a}$ Philippe F. Smet, (D) ${ }^{\mathrm{b}}$ Lisa I. D. J. Martin, (D) ${ }^{\mathrm{b}}$ Christian Pritzel $^{\mathrm{C}}$ and \\ Jörn Schmedt auf der Günne (iD *a
}

\begin{abstract}
Doping homogeneity is important for the properties of co-doped phosphors, as it can affect the energy transfer between sensitizer and activator ions. In a case study we apply different methods, that is scanning electron microscopy (SEM) combined with energy dispersive X-ray spectroscopy (EDX) mapping, SEM combined with cathodoluminescence $(C L)$ and solid-state nuclear magnetic resonance (NMR), to study the doping homogeneity of the host system monazite $\mathrm{LaPO}_{4}$ doped with two different lanthanide ions on different length scales. A new criterion for doping heterogeneity in co-doped systems is developed, which is based on the NMR visibility function, which for this purpose is extended to doping with two or more paramagnetic dopants. A deviation from this function is indicative of doping heterogeneity on the length-scale of the blind-spheres of the paramagnetic dopants. A discussion of the advantages and disadvantages of the different methods is presented. The combined approach allows to study doping homogeneity from the $\mathrm{nm}$ to the $\mu \mathrm{m}$ scale.
\end{abstract}

\section{Introduction}

Co-doping is often utilized to achieve extra performance for functional materials. For example, defect populations can be manipulated by co-doping in semi-conductors ${ }^{1}$ to achieve changes in conductivity or in luminescence materials to achieve higher efficiency, ${ }^{2}$ brightness or longer afterglow. ${ }^{3}$ For glass lasers ${ }^{4}$ co-doping makes a higher dopant-solubility and thermal tolerance possible. Not only the choice of suitable pairs of co-dopants needs to be considered depending on the application scenario, but also the distribution of the co-dopants in the host material is important. Co-doped afterglow materials for example critically depend on energy transfer ${ }^{5,6}$ processes between different dopant ions which are limited by their distance. As an example, the addition of $\mathrm{Dy}^{3+}$ to the $\mathrm{SrAl}_{2} \mathrm{O}_{4}: \mathrm{Eu}^{2+}$ persistent phosphor increases the brightness and the duration of the afterglow. ${ }^{7}$ However, partial segregation of dysprosium has been observed at grain boundaries, affecting the actual dopant concentration inside the phosphor particles. ${ }^{8}$

In general the concept homogeneity depends on a length scale or a volume. ${ }^{9}$ For example a glass sample may appear

\footnotetext{
${ }^{a}$ Inorganic Materials Chemistry, University of Siegen, Adolf-Reichwein-Str. 2, 57076 Siegen, Germany. E-mail: gunnej@chemie.uni-siegen.de

${ }^{b}$ Department of Solid State Sciences, Ghent University, Krijgslaan 281-S1, 9000 Gent, Belgium. E-mail: Philippe.Smet@UGent.be

${ }^{c}$ Construction and Materials Chemistry, University of Siegen, Paul-Bonatz-Str. 9-11, 57076 Siegen, Germany

$\dagger$ Electronic supplementary information (ESI) available. See DOI: 10.1039/ с9ср05599a
}

homogeneous when investigated by visible light but heterogeneous on an atomic scale. In case of co-doped phosphors the interesting length scale is of the order of the energy transfer distance (up to a few nm, depending on the transfer mechanism). In the present case we refer to "homogeneous doping", if the La atoms in the host material are statistically replaced by the lanthanides (Ln) used for doping. While different techniques are able to elucidate the distribution of dopants in the host matrix on different length scale, it turns out that it is not easy to "prove" doping homogeneity. Instead the usual approach is to disprove heterogeneity on different length scales. Analytical methods can establish heterogeneity in different ways: X-ray diffraction (XRD) as a bulk technique can be used to establish heterogeneity by deviation of the lattice parameters from Vegard's rule ${ }^{10}$ or by the appearance of a non-statistical pair distribution function ${ }^{11}$ given that the scattering contrast is sufficient. X-ray photoelectron spectroscopy (XPS) offers information about the dopant distribution at the surface on the nm scale. ${ }^{12}$ Methods based on electron microscopy can prove heterogeneity at a specific location in the sample but not in the bulk. For example, transmission electron microscopy can provide line-scans showing a dopant distribution profile on the nm scale for thin sample layers. ${ }^{13}$ Energy dispersive X-ray (EDX) mapping in a scanning electron microscope (SEM) can show heterogeneous dopant distributions on the sub- $\mu \mathrm{m}$ scale $\mathrm{e}^{14,15}$ at a sample surface to a depth of several $\mu \mathrm{m}$, depending on the accelerator voltage ${ }^{13}$ (see below). EDX is considered to be a semi-quantitative analysis technique, as the number of detected X-rays depends on the accelerating voltage, the sample composition, the detection angle 
and the sample morphology, with especially the latter effect not being easy to correct for. Cathodoluminescence (CL) imaging evaluates the spatial and spectral distribution of the emitted light after excitation by the electron beam. For a certain fixed position of the electron beam, light is generated in a similar volume compared to the characteristic X-rays. The advantage over EDX is an increased sensitivity, allowing to study lower impurity concentrations. Finally, the luminescence characteristics of many impurities depend on the local environment. Hence, the shape of the CL emission spectrum can be used to probe the local chemical composition. ${ }^{16,17}$ Electron paramagnetic resonance (EPR) studies the signals from paramagnetic dopants in low concentration and extracts information on distribution as well as local environment ${ }^{18}$ from spectroscopic parameters such as the lineshape. ${ }^{19} \mathrm{~A}$ broad linewidth is typical for strong couplings and indicative of heterogeneity. ${ }^{20}$

In this contribution solid-state NMR is used to investigate co-doping heterogeneity. In case of a diamagnetic solid host with NMR-active nuclei solid-state NMR has been used to investigate the dopant distribution of a single kind of paramagnetic dopant ("mono-doped") in different ways: spin-lattice $T_{1}$ relaxation, ${ }^{21-23}$ line width ${ }^{24-26}$ and visibility function (see below). ${ }^{27,28}$ A $T_{1}-T_{2}$ correlation map was also shown to be useful in studying a paramagnetic site distribution at the surface of porous materials. ${ }^{29}$ These NMR observables of the host-material are influenced by the hyperfine interaction ${ }^{30,31}$ between the unpaired electrons of a paramagnetic dopant and the NMR-active nucleus in a non-trivial way in general. For Ln(III) dopants the valence electrons are situated in f-orbitals for which the spin-orbit (SO) interactions dominate over ligand field effects as compared to transition metal atoms for which the order is reversed. ${ }^{32}$ Thus the magnetism of Ln(III) ions can be predicted neglecting ligand field effects in the high-temperature regime with the exception of $\mathrm{Sm}$ (III) and $\mathrm{Eu}(\mathrm{III})$. In fact the NMR visibility function, the relaxation behavior and the line-width of host signals have been shown to correlate in a simple way with an increasing $\operatorname{Ln}$ (III) doping concentration for the host chosen here. ${ }^{26}$ Here we make use of the NMR visibility, which is the molar peak area of NMR signal of a paramagnetically doped compound normalized by that of the undoped host. The visibility function is the NMR visibility as a function of doping concentration that can be approximated analytically by a decaying exponential function, if it is assumed that around every paramagnetic dopant a certain volume exists within which NMR nuclei become "invisible" to the NMR experiment. Invisible here means that signals decay very fast so that they do not contribute to the FID which is sampled after the spectrometer deadtime. This concept was independently introduced by different groups in the context of dynamic nuclear polarization ${ }^{33}$ and for the purpose of studying doping homogeneity. ${ }^{27}$ It could be shown that in a homogeneous system the visible percentage of the host NMR signal decays in an exponential fashion with increasing doping concentration which can be used to estimate the radius of the blind sphere around a paramagnetic center (formula see experimental part). ${ }^{26,27}$ In mono-doped materials deviations from this exponential function may serve as a criterion of doping heterogeneity in the above sense on the length scale of the blind-sphere radius ${ }^{26-28}$ which typically takes values up to $\sim 2 \mathrm{~nm}$. While in general it may not always be trivial to explain what contributes to the blind sphere radius it appears that for $\operatorname{Ln}(\mathrm{III})$ dopants the pseudo-contact shielding is strongly correlated with the size of the blind sphere. ${ }^{26}$ Correspondingly the blind-spheres for different NMR active nuclei in the same material are related to the size of the gyromagnetic ratio. $^{26}$ The only known exception for $\operatorname{Ln}($ III) dopants is $\mathrm{Gd}$ (III) which is known for its very special relaxation behavior which also makes it an effective contrast agent in magnetic resonance imaging. ${ }^{31}$

Given the interest in co-doped luminescent materials the target of this study is to investigate whether the NMR visibility function can be extended to co-doped systems and to compare this approach to other commonly used techniques to investigate doping homogeneity.

\section{Experimental}

Reference materials for co-doped monazite $\mathrm{LaPO}_{4}$ were obtained with a co-precipitation method and subsequent annealing which previously had been shown to produce homogeneously mono-doped samples. ${ }^{26,28}$ The starting reagents, which include $\mathrm{La}_{2} \mathrm{O}_{3}$ (ChemPur, 99.99\%) and dopant materials $\mathrm{Gd}_{2} \mathrm{O}_{3}$ (smart elements, 99.99\%), $\mathrm{Dy}_{2} \mathrm{O}_{3}$ (smart elements, 99.999\%), $\mathrm{Ho}_{2} \mathrm{O}_{3}$ (smart elements, 99.99\%), $\mathrm{Yb}_{2} \mathrm{O}_{3}$ (smart elements, 99.99\%) and $\mathrm{Nd}_{2} \mathrm{O}_{3}$ (ChemPur, 99.99\%), were mixed and dissolved in the required ratios in concentrated nitric acid. Upon adding $\mathrm{NH}_{4} \mathrm{H}_{2} \mathrm{PO}_{4}$ (VWR chemicals) solution in excess a precipitate is formed, washed with water and ethanol, then centrifuged and dried overnight. Annealing is done in corundum crucibles at $1000{ }^{\circ} \mathrm{C}$ for $4 \mathrm{~h}$.

Solid state NMR measurements were performed on a Bruker Avance II spectrometer at 7.05 T. Magic angle spinning (MAS) was done with $4 \mathrm{~mm}$ pencil rotors at $10 \mathrm{kHz}$ with a home-built McKay probe head and a dead-time delay of $15 \mu \mathrm{s}$. External quantification of the NMR signals was assisted with a microbalance (Sartorius MC5). The NMR visibility $f$ was calculated as the observed peak area of the ${ }^{31} \mathrm{P}$ NMR signal per mole of the sample normalized by the peak area per mole of non-doped diamagnetic host. Deconvolution of NMR peaks was done with the program deconv2Dxy. ${ }^{34}$ The NMR visibility fitting function ${ }^{27,28}$ for a homogeneously mono-doped sample is defined as $f(x)=\exp \left(-a r_{0}^{3} x\right)$, where $r_{0}$ is the blind-sphere radius, $a$ is a parameter related to the number density of dopable sites ( $a=4 / 3 \pi N_{\text {hostUC }} / V_{\mathrm{UC}}$ ), $N_{\text {hostUC }}$ is the number of "dopable" sites in the unit cell, $x$ is the fraction of atoms substituted by a paramagnetic dopant (see example) and $V_{\mathrm{UC}}$ is the volume of the unit cell. For example, for monazite ${ }^{35}$ doped with Dy, i.e. $\mathrm{LaPO}_{4}$ :Dy or $\mathrm{La}_{1-x} \mathrm{Dy}_{x} \mathrm{PO}_{4}, a$ is $0.055 \AA^{-3}$.

Scanning electron microscopy images were obtained in back-scattered electron detection mode (Hitachi S-3400 N). For elemental mapping with EDX, the connected ThermoScientific Noran System 7 was used. CL radiation emitted by the sample was collected for a 256-by-184 grid (dwell time of $100 \mathrm{~ms}$ per point) with an optical fiber and delivered to an Acton SP2300 monochromator 
and ProEM 1600EMCCDcamera (Princeton Instruments). CL spectra and intensities were processed off-line. All spectra were measured at room temperature and with an accelerating voltage of $20 \mathrm{kV}$. To avoid electrical charging of the sample, and the associated image deformations, a low background pressure of $20 \mathrm{~Pa}$ was used. For EDX quantification of the lanthanide ion concentrations, the L lines were monitored (La: $4.7 \mathrm{keV}, \mathrm{Nd}$ : $5.2 \mathrm{keV}$, Tm: $7.2 \mathrm{keV})$.

\section{Results and discussion}

To investigate the effects of co-doping on the NMR visibility function, reference compounds are required which are homogeneously doped. To this end $\mathrm{LaPO}_{4}$ was co-doped with $\mathrm{Nd} / \mathrm{Tm}$, $\mathrm{Gd} /$ Dy and $\mathrm{Nd} / \mathrm{Ho}$ at different concentrations.

The synthesis recipe had been used before ${ }^{26,28}$ to produce single-doped monazite which proved to be homogeneous according to XRD and NMR with respect to the Vegard behavior of the lattice parameters and the NMR visibility function, ${ }^{27}$ respectively. In the following the doping homogeneity of co-doped $\mathrm{LaPO}_{4}$ is first tested with energy dispersive X-ray mappings and cathodoluminescence. Finally it is shown how the NMR visibility function needs to be modified for application to co-doped materials.

\section{Doping homogeneity according to EDX}

Monazite $\mathrm{LaPO}_{4}$ is obtained by dehydration of rhabdophane $\mathrm{LaPO}_{4} \cdot \mathrm{H}_{2} \mathrm{O}$ which in turn is obtained by precipitation from a solution containing lanthanum and monophosphate ions. Depending on washing steps and how fast the precipitation is conducted, impurities by hydrogen phosphates can be observed which after high temperature annealing convert into $\mathrm{LaP}_{3} \mathrm{O}_{9} \cdot{ }^{36}$ This impurity (less than 5\% based on NMR peak area quantification results) should not have an influence on the distribution of the lanthanide dopants but may cause the formation of grain boundaries and could give rise to deviations in electron microscopy which is sensitive to the surface of the particles. Powder $\mathrm{X}$-ray diffraction (not shown) indicates a very small impurity of $\mathrm{LaP}_{3} \mathrm{O}_{9}$ which is consistent with ${ }^{31} \mathrm{P}$ MAS NMR. Other side phases could not be observed. Nevertheless, given that solubility limits for a solid solution of $\mathrm{LaPO}_{4}: \mathrm{Ln}$ exist ${ }^{37}$ and that light lanthanides prefer to crystallize in the monazite structure type but heavy lanthanides prefer to crystallize in the zircon structure type, it is reasonable to further investigate the distribution of lanthanide dopants.

Energy dispersive X-ray spectroscopy (EDX) in comparison to $\mathrm{XRD}$ is a local technique which elucidates sample composition by the emitted X-ray spectrum inside the excitation volume of the electron beam, which at $20 \mathrm{keV}$ amounts to a 1-2 micrometers in $\mathrm{LaPO}_{4}$. In electron micrographs and EDX maps (Fig. 1 and Fig. S1-S3, ESI $\dagger$ ) grain boundaries with different composition in terms of the $\mathrm{Ln} / \mathrm{P}$ ratio could be observed but apparently the total amount was too small to have an impact on the NMR experiments (see below). The element composition ratios for the different lanthanides reflect the batch composition of the starting

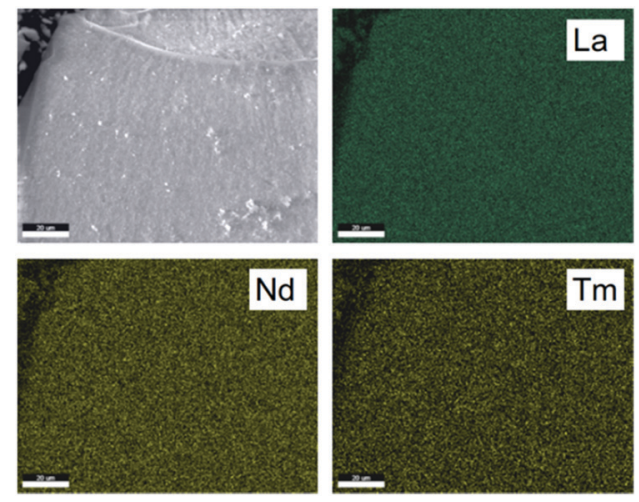

Fig. 1 Electron micrographs detected via backscattered electrons (top left) and EDX mappings (top right and bottom) of $\mathrm{La}_{0.8} \mathrm{Nd}_{0.1} \mathrm{Tm}_{0.1} \mathrm{PO}_{4}$. The acceleration voltage is $30 \mathrm{kV}$.

materials and are consistent with a homogeneous distribution throughout the material, at the sub-micron length scale. To assess the spatial resolution which could potentially be obtained in the studied material system, Monte Carlo simulations using Casino ${ }^{38,39}$ have been performed, where the interactions of the incoming electrons with the sample are simulated, including the creation and eventual detection of characteristic X-rays. The chosen electron energy was $20 \mathrm{keV}$, as a compromise between EDX signal intensity and spatial resolution. Indeed, lowering the electron beam energy reduces the interaction volume, but simultaneously reduces the number of generated X-rays. Specifically for the studied $\mathrm{LaPO}_{4}: \mathrm{Ln}$ system, the L-lines of the lanthanides are monitored, in order to avoid the strongly overlapping M-lines, which require an electron beam energy of at least $15 \mathrm{keV}$ to have sufficient yield. Given the low concentrations (and thus also the corresponding EDX signal intensities) of the dopants with respect to the La present in the host, this is an experimental constraint. Consequently, it is not obvious what spatial resolution can be obtained if one wants to assess the dopant homogeneity in the co-doped $\mathrm{LaPO}_{4}$ samples. Therefore, $\mathrm{La}_{1-x}(\mathrm{Nd}, \mathrm{Tm})_{x} \mathrm{PO}_{4}$ was simulated as a model system (Fig. 2), where the doping was considered fully inhomogeneous.

A slab of $\mathrm{La}_{0.99} \mathrm{Tm}_{0.01} \mathrm{PO}_{4}$ was inserted in between two slabs consisting of $\mathrm{La}_{0.99} \mathrm{Nd}_{0.01} \mathrm{PO}_{4}$. The width of the central $\mathrm{Tm}$ doped slab was varied from $100 \mathrm{~nm}$ to $2 \mu \mathrm{m}$, while the (vertical) thickness was taken larger than the penetration depth of the electron beam. Then the electron beam was swept over the sample surface, across the three slabs. For each simulated point of incidence, the obtained EDX signal intensities for $\mathrm{Nd}$ and Tm were stored and plotted (Fig. 2). While the lanthanide concentrations follow a step like function, the concentration profile is smoothed by the interaction volume in the sample. For the widest central slab of $2 \mu \mathrm{m}$, the concentration profiles show that distinct regions can be found, where e.g. no X-rays originating from $\mathrm{Nd}$ are found. For narrower central slabs, a further smoothing occurs, and already at a central width of $1 \mu \mathrm{m}, \mathrm{Nd}$ is measured along the entire profile. When the central Tm-doped slab is only $100 \mathrm{~nm}$ wide, the Nd signal only drops by about $20 \%$ in the middle of the Tm-doped slab, as compared to positions far away from the central slab. Taking into account 


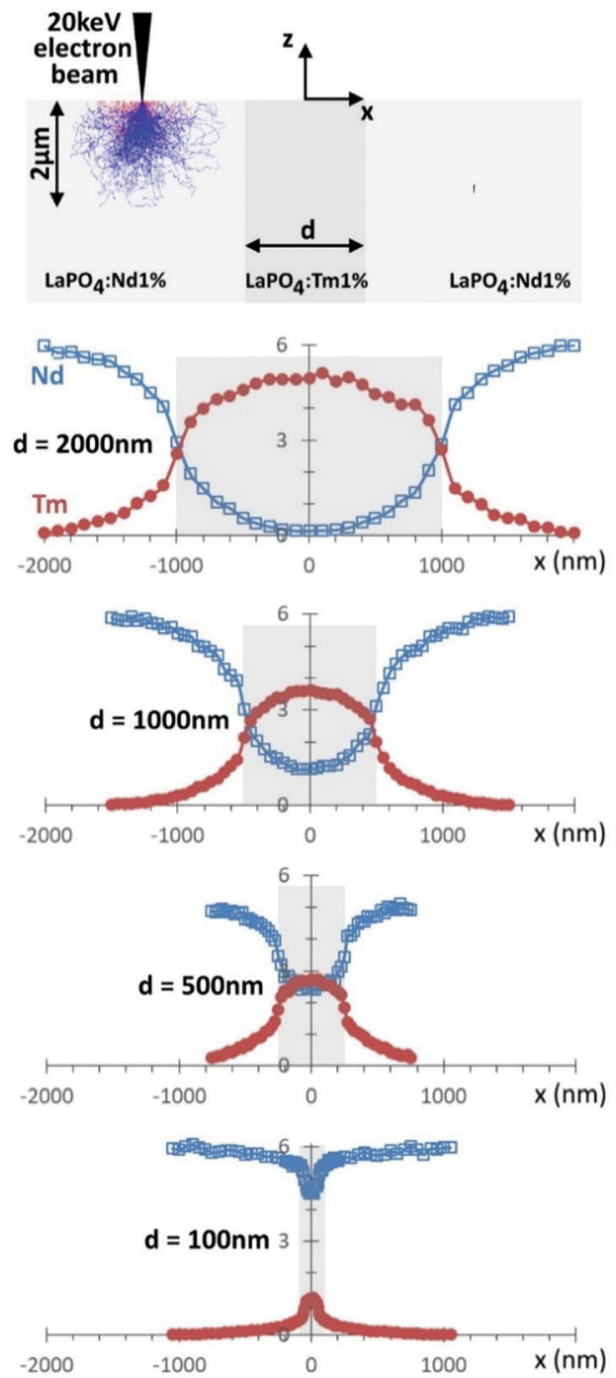

Fig. 2 Monte Carlo simulations of the EDX signal intensities of $\mathrm{Nd}$ and $\mathrm{Tm}$ were performed for a specific geometry of three slabs next each other, with the outer ones consisting of $\mathrm{La}_{0.99} \mathrm{Nd}_{0.01} \mathrm{PO}_{4}$ and the central one, with thickness $d$, being $\mathrm{La}_{0.99} \mathrm{Nd}_{0.01} \mathrm{PO}_{4}$ (see top schematic, which also includes the underlying electron paths of a typical simulation). The derived concentration profiles along the $x$ direction are shown for different thicknesses $d$.

the experimental constraints, where the Nd EDX signal will be low given the low doping concentration, the noise on each measured point will likely obscure this small dip in the concentration profile. Similarly, while there is for the central Tm-doped slab of $100 \mathrm{~nm}$ a clear increase in the Tm concentration when crossing this slab, the signal intensities will be fairly low (e.g. as when compared to the $2 \mu \mathrm{m}$ wide slab), because the electrons spread laterally out into the $\mathrm{Nd}$-doped slabs. Consequently, mapping of the dopant concentration by EDX will - under the mentioned constraints of signal intensity - not allow to quantitatively assess the dopant homogeneity on the submicron, or smaller, scale. Of course, if the (vertical) thickness of the sample can be reduced by preparing a thin slab, the spatial resolution increases, as the electrons leave the sample on the bottom side before they can spread too much laterally.

\section{Fluorescence spectroscopy}

When the electron beam hits the sample, also cathodoluminescent radiation is generated in a similar volume as the characteristic X-rays, used for the EDX analysis. The advantage is however that the CL emission can be strong, translating in a high signal intensity, and that the contributions in the emission spectrum can be related to specific luminescent ions, especially in the case of the $4 \mathrm{f}-4 \mathrm{f}$ emitting trivalent lanthanides. In principle, the emission intensity can be related to the local dopant concentration as there is a linear relation between both, until concentration quenching sets in, where the CL intensity increases sublinearly (or even reduces) for increasing dopant concentration.

The CL emission spectra of codoped $\mathrm{La}_{0.984} \mathrm{Nd}_{0.008} \mathrm{Tm}_{0.008} \mathrm{PO}_{4}$ and $\mathrm{La}_{0.968} \mathrm{Nd}_{0.016} \mathrm{Tm}_{0.016} \mathrm{PO}_{4}$, together with those for single doped $\mathrm{La}_{0.995} \mathrm{Nd}_{0.005} \mathrm{PO}_{4}$ and $\mathrm{La}_{0.995} \mathrm{Tm}_{0.005} \mathrm{PO}_{4}$, have been measured and compared (Fig. 3). Those spectra were acquired while the electron beam was continuously swept over a large area $(100 \mu \mathrm{m} \times 100 \mu \mathrm{m})$ to find the average emission spectrum. The emission of $\mathrm{La}_{0.995} \mathrm{Nd}_{0.005} \mathrm{PO}_{4}$ is dominated by the transitions in the infrared (with the ${ }^{4} \mathrm{~F}_{3 / 2} \rightarrow{ }^{4} \mathrm{I}_{9 / 2}$ transitions around $900 \mathrm{~nm}$ ), accompanied by several transitions in the visible part of the spectrum, originating from the higher-lying ${ }^{2} \mathrm{~F}(2)_{5 / 2},{ }^{4} \mathrm{D}_{3 / 2}$ and ${ }^{2} \mathrm{P}_{3 / 2}$ levels. ${ }^{40}$ For $\mathrm{La}_{0.995} \mathrm{Tm}_{0.005} \mathrm{PO}_{4}$, the main emission bands are around $345 \mathrm{~nm}$ $\left({ }^{3} \mathrm{P}_{0} \rightarrow{ }^{3} \mathrm{~F}_{4}\right), 360 \mathrm{~nm}\left({ }^{1} \mathrm{D}_{2} \rightarrow{ }^{3} \mathrm{H}_{6}\right), 455 \mathrm{~nm}\left({ }^{3} \mathrm{P}_{0} \rightarrow{ }^{3} \mathrm{H}_{4},{ }^{1} \mathrm{D}_{2} \rightarrow{ }^{3} \mathrm{~F}_{4}\right)$, $475 \mathrm{~nm}\left({ }^{1} \mathrm{G}_{4} \rightarrow{ }^{3} \mathrm{H}_{6}\right), 650 \mathrm{~nm}\left({ }^{1} \mathrm{G}_{4} \rightarrow{ }^{3} \mathrm{~F}_{4}\right)$ and from 700 to $800 \mathrm{~nm}$ $\left({ }^{3} \mathrm{~F}_{2},{ }^{3} \mathrm{~F}_{3},{ }^{3} \mathrm{H}_{4} \rightarrow{ }^{3} \mathrm{H}_{6}\right) \cdot{ }^{41}$ No emission peaks related to other lanthanides were found, pointing at good purity of the prepared phosphors. The CL emission spectrum of the Nd, Tm co-doped $\mathrm{La}_{0.984} \mathrm{Nd}_{0.008} \mathrm{Tm}_{0.008} \mathrm{PO}_{4}$ is essentially the sum of the contributions of both $\mathrm{Tm}^{3+}$ and $\mathrm{Nd}^{3+}$ ions (Fig. 3c), although subtle differences can be spotted as function of the doping concentration, e.g. in the relative intensity of the $\mathrm{Tm}^{3+}$ transitions at $345 \mathrm{~nm}$ and $360 \mathrm{~nm}$ which originate from ${ }^{3} \mathrm{P}_{0}$ and ${ }^{1} \mathrm{D}_{2}$, respectively. To assess the degree of energy transfer between $\mathrm{Tm}^{3+}$ and $\mathrm{Nd}^{3+}$, the dopant

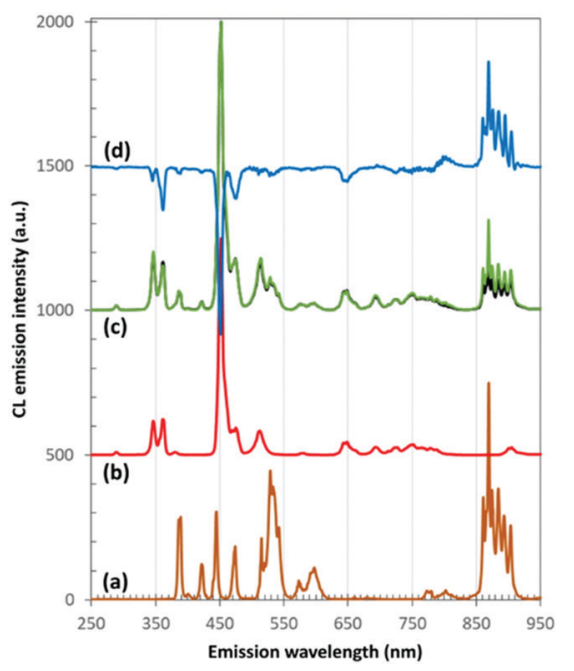

Fig. 3 The average $C L$ emission spectra of (a) $\mathrm{La}_{0.995} \mathrm{Nd}_{0.005} \mathrm{PO}_{4}$ and (b) $\mathrm{La}_{0.995} \mathrm{Tm}_{0.005} \mathrm{PO}_{4}$. (c) CL emission spectra of $\mathrm{La}_{0.984} \mathrm{Nd}_{0.008} \mathrm{Tm}_{0.008} \mathrm{PO}_{4}$ (black) and $\mathrm{La}_{0.968} \mathrm{Nd}_{0.016} \mathrm{Tm}_{0.016} \mathrm{PO}_{4}$ (green). (d) Difference between both spectra in (c), multiplied by a factor of five. 
concentration of both $\mathrm{Tm}$ and $\mathrm{Nd}$ was doubled (i.e. the phosphor with composition $\mathrm{La}_{0.968} \mathrm{Nd}_{0.016} \mathrm{Tm}_{0.016} \mathrm{PO}_{4}$, Fig. 3c). This doesn't dramatically alter the emission spectrum and to better appreciate the differences, the difference spectrum between both co-doped samples is shown in Fig. 3d.

For $\mathrm{La}_{0.968} \mathrm{Nd}_{0.016} \mathrm{Tm}_{0.016} \mathrm{PO}_{4}$, the $\mathrm{Nd}^{3+}$ transitions in the near IR between 850 and $920 \mathrm{~nm}$ increase in relative intensity, while the $\mathrm{Nd}^{3+}$ transitions in the visible are slightly reduced, compared to $\mathrm{La}_{0.984} \mathrm{Nd}_{0.008} \mathrm{Tm}_{0.008} \mathrm{PO}_{4}$. For the $\mathrm{Tm}^{3+}$ emission lines, most of the transitions below $675 \mathrm{~nm}$ reduce in intensity, where especially the transitions originating from ${ }^{1} \mathrm{D}_{2}$ reduce in intensity, which could be due to increased cross-relaxation in $\mathrm{Tm}^{3+}$ or to increased energy transfer to $\mathrm{Nd}^{3+}$, as the $\mathrm{Tm}^{3+}\left({ }^{1} \mathrm{D}_{2}\right)$ level is at approximately the same energy as the $\mathrm{Nd}^{3+}\left({ }^{4} \mathrm{D}_{3 / 2}\right)$ level with respect to their ground states.

Of course, the CL spectra discussed above are only average CL spectra. Therefore, SEM-CL-EDX mapping was used to probe the distribution of both dopant ions on the submicron scale, where for every pixel the full emission spectrum was recorded in the range from 400 to $920 \mathrm{~nm}$. To assess the doping homogeneity, the barycenter emission wavelength $\lambda_{\mathrm{bc}}$

$$
\lambda_{\mathrm{bc}}=\frac{\int_{400 \mathrm{~nm}}^{910 \mathrm{~nm}} I(\lambda) \lambda \mathrm{d} \lambda}{\int_{400 \mathrm{~nm}}^{910 \mathrm{~nm}} I(\lambda) \mathrm{d} \lambda}
$$

was calculated for the measured spectral window (Fig. 4). Given that the value of $\lambda_{\mathrm{bc}}$ changes from $542 \mathrm{~nm}$ for $\mathrm{La}_{0.995} \mathrm{Tm}_{0.005} \mathrm{PO}_{4}$ to $694 \mathrm{~nm} \mathrm{La} \mathrm{La}_{0.995} \mathrm{Nd}_{0.005} \mathrm{PO}_{4}$, any change in the relative contribution by both dopants to the total emission spectrum will readily be reflected. This approach is valid as for the studied concentrations the concentration quenching is limited, as well as the energy transfer between the dopant ions. For instance, the barycenter wavelength increases only $10 \mathrm{~nm}$ when going from $\mathrm{La}_{0.984} \mathrm{Nd}_{0.008} \mathrm{Tm}_{0.008} \mathrm{PO}_{4}$, to $\mathrm{La}_{0.968} \mathrm{Nd}_{0.016} \mathrm{Tm}_{0.016} \mathrm{PO}_{4}$. The map
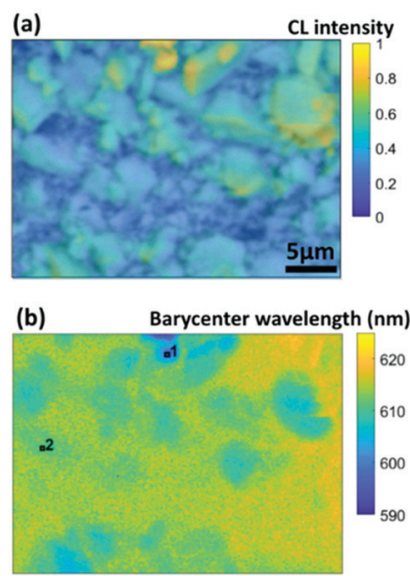

(c)

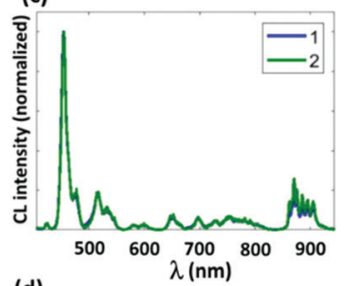

(d)

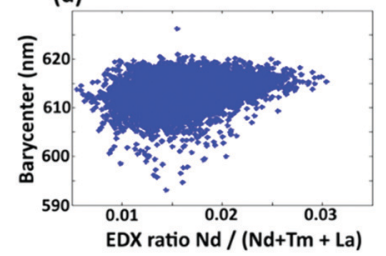

Fig. 4 (a) SEM image of $\mathrm{La}_{0.968} \mathrm{Nd}_{0.016} \mathrm{Tm}_{0.016} \mathrm{PO}_{4}$ obtained by the backscattered electrons, with the color overlay showing the integrated $\mathrm{CL}$ intensity per pixel in the $256 \times 192$ sampled grid. (b) Barycenter wavelength. (c) Two local CL emission spectra as measured in the two pixels indicated in the barycenter map (b). (d) Scatter plot of the barycenter wavelength as function of the Nd EDX signal intensity, normalized to the lanthanide EDX signal intensity. shows that the barycenter wavelength is narrowly distributed (Fig. 4b). For instance, no phosphor particles with dominating $\mathrm{Tm}^{3+}$ or $\mathrm{Nd}^{3+}$ emission were found. Apart from a few small particles with $\lambda_{\mathrm{bc}}$ in the range from 590 to $600 \mathrm{~nm}$ (spectrum 1 in Fig. 4c), for most measured points a $\lambda_{\mathrm{bc}}$ between 605 and $620 \mathrm{~nm}$ is found. Comparing the CL intensity and topography map in Fig. 4a with the $\lambda_{\text {bc }}$ map in Fig. 4 b, there appears a strong correlation between $\lambda_{\mathrm{bc}}$ and the CL intensity. Bigger and smoother particles, protruding from the sample surface, have slightly shorter barycenter wavelength. This is likely related to a lower fraction of reabsorption of emitted light or by a reduced optical path length due to limited scattering, in comparison to lower lying, smaller particles. Clearly, when considering only the bigger particles, the variation on the barycenter wavelength is no more than a few nm. For comparison, a scatter plot was made (Fig. 4d) where the relative EDX intensity of Nd compared to all lanthanides is plotted versus $\lambda_{\mathrm{bc}}$. No clear trend can be found, although the Nd concentration appears to slightly increase for longer barycenter wavelengths, which is not unexpected based on the spectral positions of the $\mathrm{Tm}^{3+}$ and $\mathrm{Nd}^{3+}$ emission. The low EDX signal intensities for the $\mathrm{Nd}$ (and $\mathrm{Tm}$ ) lines however introduce a great uncertainty and make this an unreliable metric to be used for dopant mapping, at least on this particular phosphor. In that sense the CL spectroscopy is far more sensitive and points at a very homogeneous distribution of both Tm and $\mathrm{Nd}$ dopants on the submicron length scale, given the sensitivity of this metric. Of course, it cannot make statements on the average $\mathrm{Nd}-\mathrm{Tm}$ distance, which is about two orders of magnitude smaller than the spatial resolution of SEM-CL on these powder samples.

\section{NMR: visibility function for paramagnetic co-doping of a diamagnetic host}

For mono-doped systems, the NMR visibility $f(x)$ for homogeneous doping shows an exponential dependence $f(x)=\exp \left(-a r_{0}^{3} x\right)$ on the doping concentration $x$, the number density parameter $a$ and the radius of the blind sphere $r_{0}$. Heterogeneity causes a deviation from the homogeneous curve into the direction of complete phase segregation. ${ }^{28}$ The curve for complete phase segregation can be estimated assuming a minimum blind sphere radius, which can be illustrated on the example of $\mathrm{LaPO}_{4}$ :Tm, i.e. $\mathrm{La}_{1-x} \mathrm{Tm}_{x} \mathrm{PO}_{4}$. Given the mixture falls apart into $(1-x) \mathrm{LaPO}_{4}$ and $x \mathrm{TmPO}_{4}$ and the NMR active nuclei in $\mathrm{TmPO}_{4}$ are "invisible" because the blind sphere is covering them, then the maximum possible visibility $f_{\max }(x)$ is approaching $f_{\max }(x)=1-x^{27}$

The exponential visibility function for mono-doped systems was deduced in two different ways for a homogeneous doping scenario. We derived it by an inductive argument, i.e. a Taylor expansion for the low-doping regime which allowed to parameterize a decaying exponential function and a numerical verification on discrete, crystalline, statistically doped structures ${ }^{27}$ by counting the numbers of atoms in- and outside the blindspheres, which also allows to qualify when the assumption of a constant dopant number density is not valid anymore. Griffin and coworkers (ref. 33, Supp. Info. p. S10), independently derived it by an elegant probabilistic ansatz. ${ }^{42}$ In the latter the idea is to calculate the probability for not finding a small voxel inside any blind-sphere 
in a given volume $V$, where the number density $n$ is the number density of blind spheres. In a given volume $V$ in total $N=n V$ blind spheres are introduced.

$$
\begin{aligned}
p_{\text {visible }} & =\lim _{N \rightarrow \infty}\left(1-\frac{V_{\text {blind }}}{V}\right)^{N}=\lim _{N \rightarrow \infty}\left(1-\frac{n \cdot V_{\text {blind }}}{N}\right)^{N} \\
& =\exp \left(-n \cdot V_{\text {blind }}\right)
\end{aligned}
$$

A typical case where this approximation breaks down is when the blind sphere radii become equal or shorter than the smallest possible distance between the detected NMR nucleus and the paramagnetic center.

It is straightforward to extend this approach to a homogeneous co-doping situation with the dopants $\mathrm{Q}$ and $\mathrm{P}$.

$$
\begin{aligned}
p_{\text {visible }} & =\lim _{N_{\mathrm{P}} \rightarrow \infty, N_{\mathrm{Q}} \rightarrow \infty}\left(1-\frac{V_{\text {blind }}^{\mathrm{P}}}{V}\right)^{N_{\mathrm{P}}} \cdot\left(1-\frac{V_{\text {blind }}^{\mathrm{Q}}}{V}\right)^{N_{\mathrm{Q}}} \\
& =\exp \left(-n_{\mathrm{P}} \cdot V_{\text {blind }}^{\mathrm{P}}-n_{\mathrm{Q}} \cdot V_{\text {blind }}^{\mathrm{Q}}\right)
\end{aligned}
$$

By expressing the blind sphere volume $V_{\text {blind }}$ with the blind sphere radius $r$ the visibility function $f(x, y)$ of a co-doped material like $\mathrm{La}_{1-x-y} \mathrm{Gd}_{x} \mathrm{Dy}_{y} \mathrm{PO}_{4}$ can be written as

$$
f(x, y)=\exp \left(-a r_{1}{ }^{3} x\right) \exp \left(-a r_{2}{ }^{3} y\right)
$$

where $r_{1}$ and $r_{2}$ are the blind sphere radii of the two dopants respectively, and $a$ is a parameter related to the number $N_{\mathrm{UC}}$ of dopable sites in the volume $V_{\mathrm{UC}}$ of the unit cell equal to the case of the mono-doped system.

$$
a=4 / 3 \pi N_{\mathrm{UC}} / V_{\mathrm{UC}}
$$

\section{Validation of the NMR visibility function for a co-doping scenario}

In the next step this model is to be tested experimentally. For this purpose three co-doped sample series have been prepared: $\mathrm{La}_{1-x-y} \mathrm{Gd}_{x} \mathrm{Dy}_{y} \mathrm{PO}_{4}, \mathrm{La}_{1-x-y} \mathrm{Nd}_{x} \mathrm{Tm}_{y} \mathrm{PO}_{4}$, and $\mathrm{La}_{1-x-y} \mathrm{Nd}_{x} \mathrm{Ho}_{y} \mathrm{PO}_{4}$. Given the approximation works it should be possible to predict the $3 \mathrm{D}$ visibility function $f(x, y)$ from the blind-sphere radii of Gd(III), Dy(III), Nd(III), Tm(III), Ho(III) obtained from mono-doped systems for the homogeneous case, which in a previous study ${ }^{26}$ were estimated to be 13.5, 12.5, 5.5, 9.0 and $10.5 \AA$, respectively.

The co-doping systems were chosen for three different reasons: first luminescence should be feasible to have another method to disprove homogeneity (see above), second the blindspheres should have a different origin, i.e. relaxation based quenching of the NMR signal leading to homogeneous broadening, as in the case of $\mathrm{Gd}(\mathrm{III})$ and inhomogeneous line-broadening as in the case of $\mathrm{Nd}(\mathrm{III}), \mathrm{Dy}(\mathrm{III}), \mathrm{Tm}(\mathrm{III})$ and $\mathrm{Ho}(\mathrm{III}),{ }^{26}$ and third the radii should differ in size.

To ease the visualization of the NMR visibility $f(x, y)$ the doping concentration of both dopants was increased by an equal amount.

$$
f(x)=\exp \left(-a r_{1}^{3} x\right) \exp \left(-a r_{2}{ }^{3} x\right)=\exp \left[-a\left(r_{1}{ }^{3}+r_{2}{ }^{3}\right) x\right]
$$

The NMR visibility can then be shown in a $2 \mathrm{D}$ plot. For $\mathrm{La}_{1-2 x} \mathrm{Gd}_{x} \mathrm{Dy}_{x} \mathrm{PO}_{4}$ (Fig. 5) and $\mathrm{La}_{1-2 x} \mathrm{Nd}_{x} \mathrm{Tm}_{x} \mathrm{PO}_{4}$ (Fig. 6) good

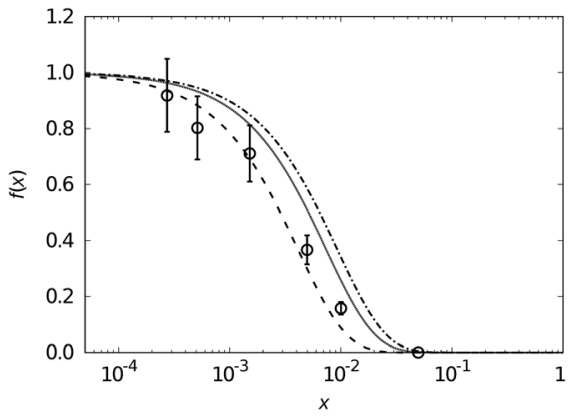

Fig. 5 NMR visibility $f(x)$ (circles with error bars) calculated from ${ }^{31} \mathrm{P}$ MAS NMR data plotted against the doping concentration $x$ of $\mathrm{La}_{1-2 x} \mathrm{Gd}_{x} \mathrm{Dy}_{x} \mathrm{PO}_{4}$ on a logarithmic scale. The dashed line features the expected visibility function $f(x)=\exp \left[-a x\left(r_{\mathrm{Gd}}{ }^{3}+r_{\mathrm{Dy}}{ }^{3}\right)\right]$ with the values $a=0.055 \AA^{-3}, r_{\mathrm{Gd}}=$ $13.5 \AA$ and $r_{\text {Dy }}=12.5 \AA$ being the same as for mono-doped $\mathrm{LaPO}_{4}$. The dotted and the dashdotted lines correspond to the $f(x)$ function neglecting Dy and Gd contribution, respectively, i.e. $f(x)=\exp \left(-\operatorname{ar}_{\mathrm{Gd}}{ }^{3} x\right)$ and $f(x)=$ $\exp \left(-\operatorname{ar}_{\mathrm{Dy}}{ }^{3} x\right)$.

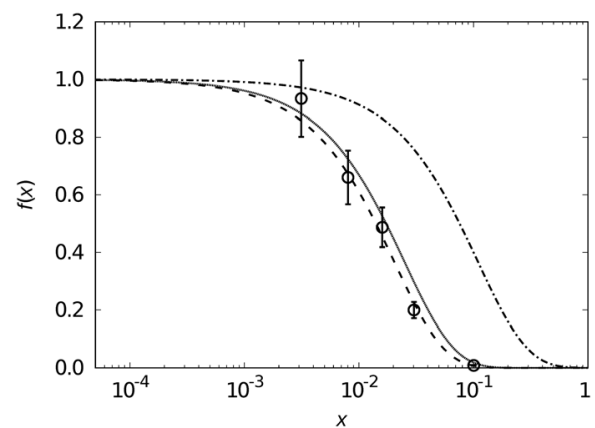

Fig. 6 NMR visibility $f(x)$ (circles with error bars) calculated from ${ }^{31} \mathrm{P}$ MAS NMR data plotted against the doping concentration $x$ of $\mathrm{La}_{1-2 x} \mathrm{Nd}_{x} \mathrm{Tm}_{x} \mathrm{PO}_{4}$ on a logarithmic scale. The dashed line features the expected visibility function $f(x)=\exp \left[-a x\left(r_{\mathrm{Nd}}{ }^{3}+r_{\mathrm{Tm}}{ }^{3}\right)\right]$ with the values $a=0.055 \AA^{-3}, r_{\mathrm{Nd}}=$ $5.5 \AA$ and $r_{\mathrm{Tm}}=9 \AA$ being the same as for mono-doped $\mathrm{LaPO}_{4}$. The dotted and the dashdotted lines correspond to the $f(x)$ function neglecting $\mathrm{Nd}$ and Tm contribution, respectively, i.e. $f(x)=\exp \left(-\operatorname{ar}_{\mathrm{Tm}}{ }^{3} x\right)$ and $f(x)=$ $\exp \left(-a r_{\mathrm{Nd}}{ }^{3} x\right)$

agreement between experimental data and the curve expected from the blind sphere radii obtained from mono-doped systems is observed, which indicates that the dopants are randomly distributed in the host onto the dopable sites.

A point which can be learned from the NMR visibility function $f(x, y)$ is that the sensitivity towards the doping concentrations $x$ and $y$ are scaled by the cubic radius of the corresponding dopants, which means that in case of a significant difference in radius like for $\mathrm{Nd}$ (III) and $\mathrm{Tm}$ (III) the bigger ion will dominate the visibility curve.

For this reason it makes sense to also test the visibility function $f(x, y)$ without imposing a linear dependence of $x$ and $y$. Such a 3D plot, the "NMR visibility map", is shown for $\mathrm{La}_{1-x-y} \mathrm{Nd}_{x} \mathrm{Ho}_{y} \mathrm{PO}_{4}$ in Fig. 7, where the contour-levels have be chosen according to the expected value for $f(x, y)$ at the point $(x, y)$. Again the experimental data showed good agreement with the assumed model. For the $\mathrm{La}_{1-x-y} \mathrm{Gd}_{x} \mathrm{Dy}_{y} \mathrm{PO}_{4}$ and $\mathrm{La}_{1-x-y} \mathrm{Nd}_{x} \mathrm{Tm}_{y} \mathrm{PO}_{4}$ series they are shown in the ESI $\dagger$ (Fig. S4 and S5). 

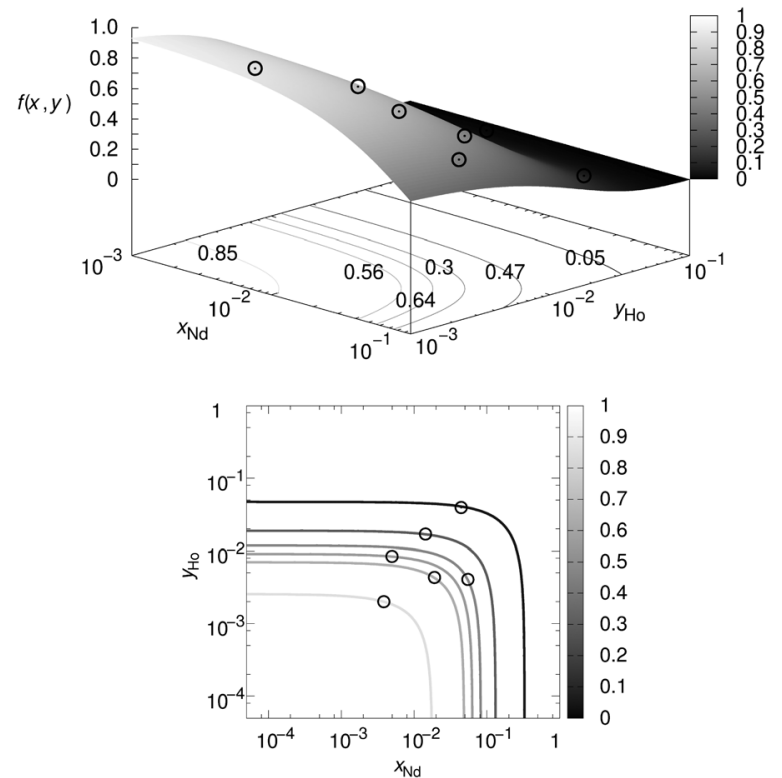

Fig. 7 NMR visibility map $f(x, y)$ calculated from ${ }^{31}$ P MAS NMR data plotted against the substitution degree $x$ and $y$ in $\mathrm{La}_{1-x-y} \mathrm{Nd}_{x} \mathrm{Ho}_{y} \mathrm{PO}_{4}$ on a logarithmic scale. The dashed 3D plot (upper) and its contour plot (lower) feature the expected visibility function $f(x, y)=\exp \left[-a\left(r_{\mathrm{Nd}}^{3} x+r_{\mathrm{Ho}}{ }^{3} y\right)\right]$ with the values $a=0.055 \AA^{-3}, r_{\mathrm{Nd}}=5.5 \AA$ and $r_{\mathrm{Ho}}=10.5 \AA$ being the same as for mono-doped $\mathrm{LaPO}_{4}$. The contour levels were chosen according to the expected value for $f(x, y)$ at the point $(x, y)$.

Which advantages and disadvantages does the NMR visibility approach have in comparison to other techniques?

An advantage is that the NMR visibility tests doping homogeneity on a length scale which is of the same order of magnitude as the critical energy transfer distance, which indicates it could have relevance to applications in luminescence.

The NMR visibility function is not directly sensitive to the distribution of the two co-dopants relative to each other on the other hand. For two co-dopants with similar blind-sphere radius, it is possible that the NMR visibility of a system where the co-dopants do not mix, looks the same as a homogeneously co-doped system. The proposed formula provides a simple criterion to test doping homogeneity in co-doped systems, nevertheless.

\section{Conclusions}

Doping homogeneity has been evaluated from $\AA$ to $\mu \mathrm{m}$ scales by a combination of different techniques: solid-state NMR, EDX and SEM-CL. $\mathrm{LaPO}_{4}$ doped with $\mathrm{Tm}^{3+}$ and/or $\mathrm{Nd}^{3+}$ was studied as a model system. For the co-doped samples, the doping homogeneity was very high at the submicron scale, as witnessed by the almost invariable emission spectrum over the evaluated sample area. It could be shown that the NMR visibility function can be predicted in case of homogeneous doping for co-doped systems given that the blind-sphere radii do not influence each other. This approximation seems to be valid in case of lanthanide dopants $\operatorname{Ln}(\mathrm{III})$. The NMR visibility function may thus be used to study doping homogeneity in co-doped systems to achieve higher light yields. The study shows that it is often necessary to apply different techniques to study doping homogeneity: bulk techniques like NMR have the advantage that a deviation from an expected ideal curve can be qualified in a simple number, while microscopic techniques with local resolution power may give qualitative evidence for heterogeneous distributions of dopants.

\section{Conflicts of interest}

There are no conflicts to declare.

\section{References}

1 J. Zhang, K. Tse, M. Wong, Y. Zhang and J. Zhu, A brief review of co-doping, Front. Phys., 2016, 11, 117405.

2 H. Kanno, Y. Hamada and H. Takahashi, Development of OLED with high stability and luminance efficiency by co-doping methods for full color displays, IEEE J. Sel. Top. Quantum Electron., 2004, 10, 30-36.

3 H. S. Jang, W. B. Im, D. C. Lee, D. Y. Jeon and S. S. Kim, Enhancement of red spectral emission intensity of $\mathrm{Y}_{3} \mathrm{Al}_{5} \mathrm{O}_{12}: \mathrm{Ce}^{3+}$ phosphor via Pr co-doping and $\mathrm{Tb}$ substitution for the application to white LEDs, J. Lumin., 2007, 126, 371-377.

4 K. Arai, H. Namikawa, K. Kumata, T. Honda, Y. Ishii and T. Handa, Aluminum or phosphorus co-doping effects on the fluorescence and structural properties of neodymiumdoped silica glass, J. Appl. Phys., 1986, 59, 3430-3436.

5 G. Blasse, Energy transfer in oxidic phosphors, Phys. Lett. A, 1968, 28, 444-445.

6 G. Blasse and B. C. Grabmaier, Luminescent Materials, Springer-Verlag, Berlin Heidelberg, 1994.

7 K. Van den Eeckhout, P. F. Smet and D. Poelman, Persistent Luminescence in $\mathrm{Eu}^{2+}$-Doped Compounds: A Review, Materials, 2010, 3, 2536-2566.

8 P. F. Smet, K. Van den Eeckhout, O. Q. De Clercq and D. Poelman, Persistent Phosphors, Handbook on the Physics and Chemistry of Rare Earths, including Actinides, Elsevier, 2015, ch. 274, vol. 48, pp. 1-108.

9 W. Horwitz, Nomenclature for sampling in analytical chemistry (Recommendations 1990), Pure Appl. Chem., 1990, 62, 1193-1208.

10 L. Vegard, Die Konstitution der Mischkristalle und die Raumfüllung der Atome, Z. Phys., 1921, 5, 17-26.

11 S. J. L. Billinge, Th. Proffen, V. Petkov, J. L. Sarrao and S. Kycia, Evidence for charge localization in the ferromagnetic phase of $\mathrm{La}_{1-x} \mathrm{Ca}_{x} \mathrm{MnO}_{3}$ from high real-space-resolution x-ray diffraction, Phys. Rev. B: Condens. Matter Mater. Phys., 2000, 62, 1203-1211.

12 T. Susi, T. Pichler and P. Ayala, X-ray photoelectron spectroscopy of graphitic carbon nanomaterials doped with heteroatoms, Beilstein J. Nanotechnol., 2015, 6, 177-192. 
13 F. Sidiroglou, A. Roberts and G. Baxter, Contributed Review: A review of the investigation of rare-earth dopant profiles in optical fibers, Rev. Sci. Instrum., 2016, 87, 041501.

14 G. Servanton, R. Pantel, M. Juhel and F. Bertin, Twodimensional quantitative mapping of arsenic in nanometerscale silicon devices using STEM EELS-EDX spectroscopy, Micron, 2009, 40, 543-551.

15 K. Van den Eeckhout, P. F. Smet and D. Poelman, Persistent luminescence in rare-earth codoped $\mathrm{Ca}_{2} \mathrm{Si}_{5} \mathrm{~N}_{8}: \mathrm{Eu}^{2+}$, J. Lumin., 2009, 129, 1140-1143.

16 T. Coenen and N. M. Haegel, Cathodoluminescence for the 21st century: Learning more from light, Appl. Phys. Rev., 2017, 4, 031103.

17 D. Poelman and P. F. Smet, Time resolved microscopic cathodoluminescence spectroscopy for phosphor research, Phys. B, 2014, 439, 35-40.

18 D. Ghica, I. D. Vlaicu, M. Stefan, V. A. Maraloiu, A. C. Joita and C. Ghica, Tailoring the Dopant Distribution in ZnO:Mn Nanocrystals, Sci. Rep., 2019, 9, 6894.

19 H. Deters, J. F. de Lima, C. J. Magon, A. S. S. de Camargo and H. Eckert, Structural models for yttrium aluminium borate laser glasses: NMR and EPR studies of the system $\left(\mathrm{Y}_{2} \mathrm{O}_{3}\right)_{0.2}-\left(\mathrm{Al}_{2} \mathrm{O}_{3}\right)_{x}-\left(\mathrm{B}_{2} \mathrm{O}_{3}\right)_{0.8-x}$, Phys. Chem. Chem. Phys., 2011, 13, 16071-16083.

20 N. Pathak, S. K. Gupta, P. S. Ghosh, A. Arya, V. Natarajan and R. M. Kadam, Probing local site environments and distribution of manganese in $\mathrm{SrZrO}_{3}: \mathrm{Mn} ; \mathrm{PL}$ and $\mathrm{EPR}$ spectroscopy complimented by DFT calculations, RSC Adv., 2015, 5, 17501-17513.

21 T. Harazono, R. Adachi, N. Kijima and T. Watanabe, ${ }^{89} \mathrm{Y}$ MAS NMR in Red Phosphor, Eu-Doped $\mathrm{Y}_{2} \mathrm{O}_{2} \mathrm{~S}$. Assignment of Peaks Shifted by Paramagnetic $\mathrm{Eu}^{3+}$, Spin Lattice Relaxation Time, and Eu Distribution, Bull. Chem. Soc. Jpn., 1999, 72, 2655-2664.

22 S. Maron, G. Dantelle, T. Gacoin and F. Devreux, NMR and ESR relaxation in $\mathrm{Nd}$ - and $\mathrm{Gd}$-doped $\mathrm{LaPO}_{4}$ : towards the accurate determination of the doping concentration, Phys. Chem. Chem. Phys., 2014, 16, 18788-18798.

23 S. Maron, N. Ollier, T. Gacoin and G. Dantelle, Determination of paramagnetic concentrations inside a diamagnetic matrix using solid-state NMR, Phys. Chem. Chem. Phys., 2017, 19, 12175-12184.

24 T. Harazono, E. Yokota, H. Uchida and T. Watanabe, ${ }^{89}$ Y-Static and MAS NMR, and ${ }^{27}$ AlMAS NMR in Green Phosphor, Tb-Doped $\mathrm{Y}_{3} \mathrm{Al}_{5} \mathrm{O}_{12}$ and Luminous Characteristics, Bull. Chem. Soc. Jpn., 1998, 71, 2797-2805.

25 T. Harazono, E. Yokota, H. Uchida and T. Watanabe, Luminous Characteristics and ${ }^{89} \mathrm{Y}$-Static NMR in Red Phosphor, Eu-Doped $\mathrm{Y}_{2} \mathrm{O}_{3}$, Bull. Chem. Soc. Jpn., 1998, 71, 825-829.

26 W. Li, Q. Zhang, J. J. Joos, P. F. Smet and J. Schmedt auf der Günne, Blind spheres of paramagnetic dopants in solid state NMR, Phys. Chem. Chem. Phys., 2019, 21, 10185-10194.
27 W. Li, V. R. Celinski, J. Weber, N. Kunkel, H. Kohlmann and J. Schmedt auf der Günne, Homogeneity of doping with paramagnetic ions by NMR, Phys. Chem. Chem. Phys., 2016, 18, 9752-9757.

28 W. Li, M. Adlung, Q. Zhang, C. Wickleder and J. Schmedt auf der Günne, A guide to brighter phosphors - linking luminescence properties to doping homogeneity probed by NMR, ChemPhysChem, 2019, 20, 3245-3250.

29 C. D'Agostino and P. Bräuer, Exploiting enhanced paramagnetic NMR relaxation for monitoring catalyst preparation using $T_{1}-T_{2}$ NMR correlation maps, React. Chem. Eng., 2019, 4, 268-272.

30 A. J. Pell, G. Pintacuda and C. P. Grey, Paramagnetic NMR in solution and the solid state, Prog. Nucl. Magn. Reson. Spectrosc., 2019, 111, 1-271.

31 I. Bertini, C. Luchinat, G. Parigi and E. Ravera, NMR of Paramagnetic Molecules, 2nd edn, Elsevier, Boston, 2017.

32 H. Lueken, Magnetochemie, ed. B. G. Teubner, Stuttgart/ Leipzig, 1999.

33 B. Corzilius, L. B. Andreas, A. A. Smith, Q. Z. Ni and R. G. Griffin, Paramagnet induced signal quenching in MAS-DNP experiments in frozen homogeneous solutions, J. Magn. Reson., 2014, 240, 113-123.

34 D. Jardón-Álvarez and J. Schmedt auf der Günne, Reduction of the temperature gradients in laser assisted high temperature MAS NMR, Solid State Nucl. Magn. Reson., 2018, 94, 26-30.

35 Y. Ni, J. M. Hughes and A. N. Mariano, Crystal chemistry of the monazite and xenotime structures, Am. Mineral., 1995, 80, 21-26.

36 C. Penot, E. Champion and P. Goursat, Synthesis and Characterisation of Lanthanum Phosphate Powders, Phosphorus Res. Bull., 1999, 10, 307-312.

37 N. Clavier, R. Podor and N. Dacheux, Crystal chemistry of the monazite structure, J. Eur. Ceram. Soc., 2011, 31, 941-976.

38 D. Drouin, A. R. Couture, D. Joly, X. Tastet, V. Aimez and R. Gauvin, CASINO V2.42-A Fast and Easy-to-use Modeling Tool for Scanning Electron Microscopy and Microanalysis Users, Scanning, 2007, 29, 92-101.

39 H. Demers, N. Poirier-Demers, A. R. Couture, D. Joly, M. Guilmain, N. de Jonge and D. Drouin, Three-dimensional electron microscopy simulation with the CASINO Monte Carlo software, Scanning, 2011, 33, 135-146.

40 M. F. Joubert, B. Jacquier, C. Linares and R. M. Macfarlane, Relaxation of the high lying states and coordination numbers of $\mathrm{Nd}^{3+}$ ions in fluorides, J. Lumin., 1992, 53, 477-482.

41 M. Quintanilla, N. O. Núñez, E. Cantelar, M. Ocaña and F. Cussó, Tuning from blue to magenta the up-converted emissions of $\mathrm{YF}_{3}: \mathrm{Tm}^{3+} / \mathrm{Yb}^{3+}$ nanocrystals, Nanoscale, 2011, 3, 1046-1052.

42 H. L. Weissberg, Effective Diffusion Coefficient in Porous Media, J. Appl. Phys., 1963, 34, 2636-2639. 